\title{
The Acoustic Spectrums of the Combustion Process in the IC-Engines
}

\author{
Ramil Shaymukhametov ${ }^{1, *}$ and Vladimir Strekalov ${ }^{1}$ \\ ${ }^{1}$ Kazan Federal University, Kremlyovskaya St., 18, Kazan, 420008, Russia
}

\begin{abstract}
The results of the Fourier spectroscopy of the acoustic noise of an internal combustion engine are presented. The sound was recorded using a voice recorders placed near the four-cylinder petrol engine. The engine speed was set to the fixed values from 900 to $3000 \mathrm{rpm}$. The distinctive characteristics of the periodograms where associated with the certain physical processes in the engine.
\end{abstract}

\section{Introduction}

The flame in a combustion chamber is an effective generator of acoustic noise. The sound energy from combustion processes comprises more than $70 \%$ of the total noise energy in a modern car.

The generation of sound itself is a complex process. The level of a harmonic of the spectrum depends not only on an oscillator, but also on various resonators. Therefore, from the spectrum of sound, one can not judge the quantitative distribution of the dynamic elements of the flame. Nevertheless, a comparative analysis of the acoustic noise spectrums under various engine operating conditions has much potential for yielding information about the combustion.

\section{Experiment technology and data processing}

The measurements were carried out on engines of various cars (Hyundai i40, $2.0 \mathrm{~L}, 150 \mathrm{hp}$; Hyundai Accent, 1.6 L., 105 hp; Chevrolet Cruze 1.6 L, 109 hp; Peugeot 4007, 2.4 L, 170 hp; Opel Astra 1.6 L, $115 \mathrm{hp}$ ). The acoustic signals were recorded using a voice recorders installed under the car's hood near the engine cylinders (in the frequency range 200-7000 $\mathrm{Hz}$, signal-to-noise ratio $37 \mathrm{~dB}$ ). The sound reference generator being located near recorders was used for calibrating. The cars stood motionless in neutral gear. The engine speed was set to the fixed values from 900 to $3000 \mathrm{rpm}$. The raw data were collected at a sampling frequency of $16 \mathrm{kHz}$ with 16-bits per sample. The spectral analysis of the data was carried out according to a standard procedure [2]. The frame length was 2048, each frame was $25 \%$ overlapped. Frames were windowed with Hamming window and thereafter FFT was applied to each frame.

\footnotetext{
* Corresponding author : ramil.shai@mail.ru
} 


\section{Experimental data and speculations}

The energy spectrums have complex line structure. Low-frequency components are missing since the microphone bandwidth began at $200 \mathrm{~Hz}$. The main energy of the acoustic noise is concentrated in the band $500-2500 \mathrm{~Hz}$ (Fig. 1).

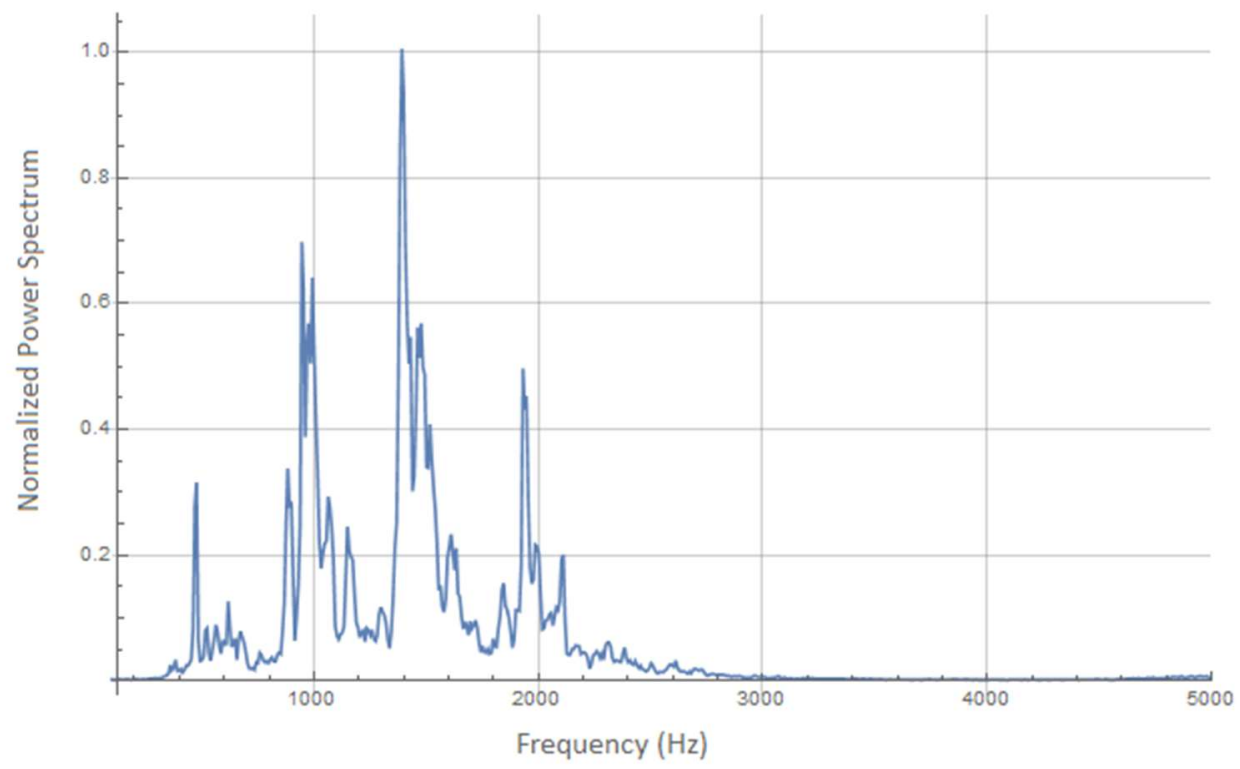

Fig. 1.Typical energy spectrum of engine noise. The crankshaft frequency is $20 \mathrm{~Hz}$ (1200 RPM).

The frequency of the maximum and spectrum width depend only weakly on the engine speed (Fig. 2). The main characteristics of the spectrums are not significantly different for various brands of cars with the same engine volume.

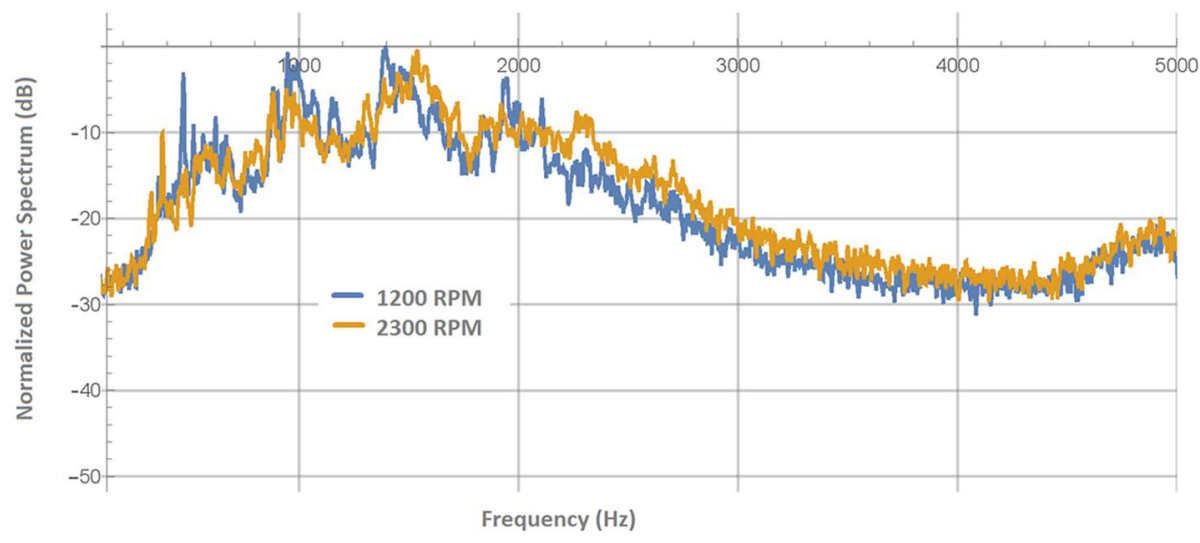

Fig. 2. Energy spectrums with a change in the frequency of rotation of the crankshaft. 
Under the hood of the car a sound reference generator was installed to calibrate the recorders. The measurements of absolute values showed a strong dependence of the noise energy on the crankshaft rotation speed. The results are depicted in Fig. 3. When the engine speed is increased by a factor of 2, the sound noise energy is increased approximately 10 times.

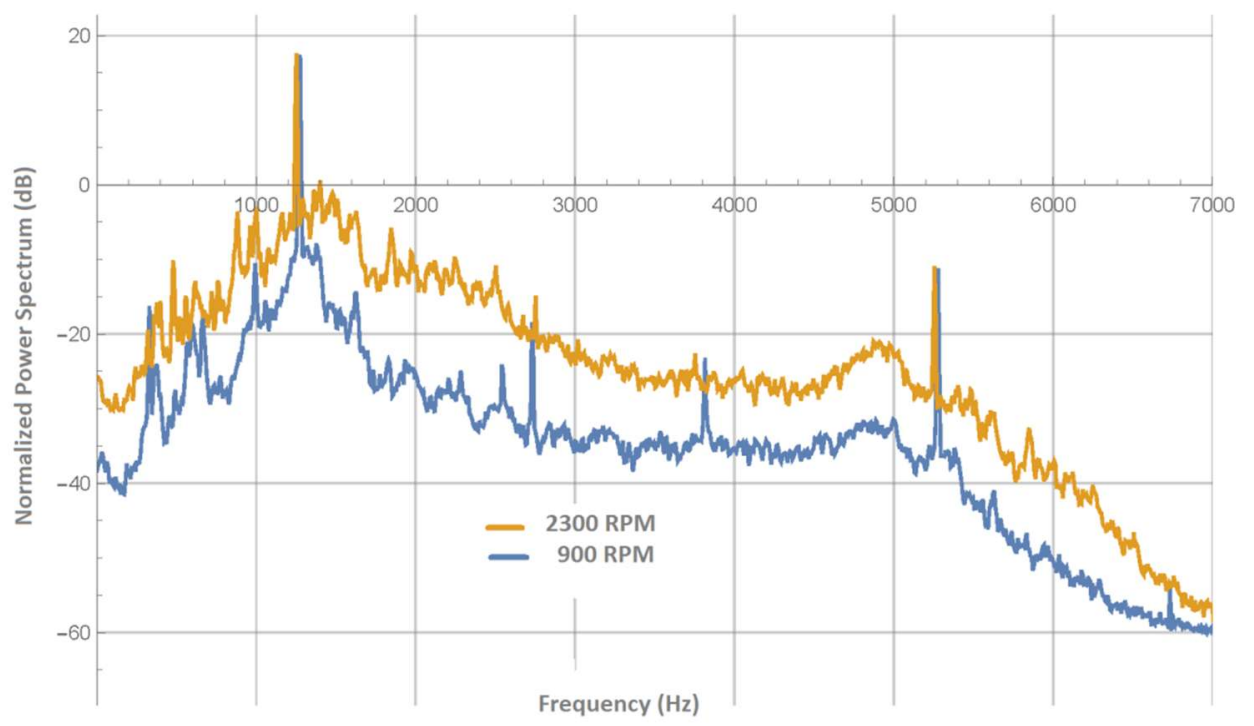

Fig. 3. The dependence of the power level on the frequency of rotation of the crankshaft.

It is likely that a cylinder volume governs the width and structure of the spectrum. Based on the flame propagation velocity in piston engines in the absence of detonation of $50-80 \mathrm{~m} / \mathrm{s}$, and the characteristic size of the combustion chamber $0.1 \mathrm{~m}$ (cylinder diameter), a lower frequency of combustion noise of 500-800 Hz can be estimated. These evaluations confirmed by observations at different volumes motor (Fig. 4). As the volume of the engine cylinder increases, the spectrum of sound becomes narrower and the distance between the peaks decreases.
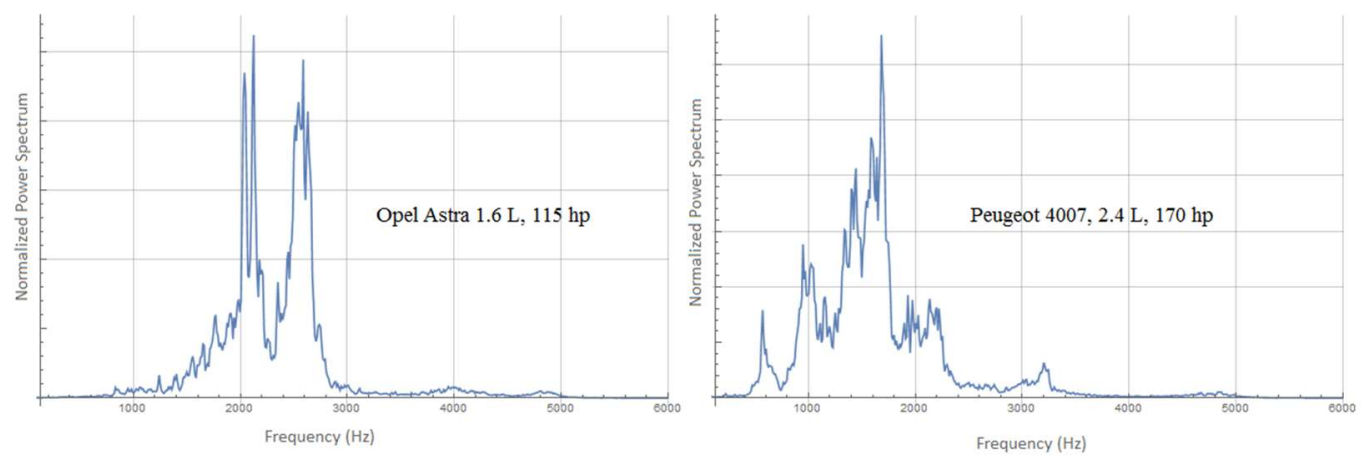

Fig. 4. Spectrums of acoustic noise at different engine volumes. 


\section{Concluding remarks}

The generation of sound is determined by complex nonlinear mechanisms, and therefore it is impossible to make a quantitative description of the combustion processes in the engine from the acoustic noise spectrum. However, comparing the spectrums under different observation conditions, one can draw qualitative conclusions about combustion as a source of sound.

The weak dependence of the spectrum characteristics on the engine speed shows that it is the combustion process that is the main source of the sound. The movements of the mechanical components of the motor only modulate the sound signal.

In the high-frequency part of the spectrum (above $4000 \mathrm{~Hz}$ ), an increased level of energy is noticeable (Fig. 4), which can be explained by the presence of detonation combustion waves. The velocity of the flame front during a detonation exceeds the speed of sound, so the detonation can generate the noise in the high-frequency part of the spectrum.

Experimental records of engine acoustic noises contain a variety of information about the dynamics of the combustion. Determination of the dependence of the spectrum characteristics on the cylinder's geometric dimensions and fuel quality is of great practical importance for the development of internal combustion engines.

\section{References}

1. R. Hickling, M.M. Kamal, Engine Noise: Excitation, Vibration, and Radiation, first ed. Springer, (1982)

2. Bendat, J. S. and Piersol, A. G. Engineering applications of correlation and spectral analysis, John Wiley \& Sons.M.(1980)

3. Withrow L, Rassweiler GM Slow motion shows knocking and nonknocking explosions. SAE Transactions 39, 297-303, 312 (1936)

4. Heywood JB. Internal combustion engine fundamentals. New York: McGraw-Hill; (1988)

5. D.E. Baxa, Noise Control in Internal Combustion Engines, Krieger Pub Co, (1989) 